\title{
COVID-19 y manifestaciones orales
}

\author{
COVID-19 and Oral Manifestations
}

\author{
Marco Cornejo Ovalle ${ }^{1,2}$ \& Iris Espinoza Santander ${ }^{3}$
}

Sr. Editor,

El coronavirus SARS-CoV-2 causa la enfermedad por coronavirus 2019 (COVID-19), una infección que produce un síndrome respiratorio agudo severo, que se ha caracterizado por fiebre, síntomas respiratorios y gastrointestinales, así como otras manifestaciones sistémicas (Guan et al., 2020).

Dado que este virus se informó oficialmente en diciembre de 2019 y se asoció con COVID-19, ha surgido literatura científica con informes de casos y manifestaciones menos frecuentes, que han esbozado mejor la historia natural y la semiología de la enfermedad de COVID-19. Sin embargo, hasta la fecha todavía hay preguntas importantes relacionadas con el enfoque clínico de la pandemia de COVID-19 y todavía hay aspectos que deben estudiarse.

En este contexto, se han ido reportado manifestaciones clínicas, que han ido enriqueciendo el conocimiento sobre las características del cuadro clínico. Uno de los aspectos escasamente documentados es la semiología a nivel de la cavidad oral. Lo que, desde nuestro punto de vista, puede deberse a que los odontólogos, a nivel mundial, se han visto separados de la actividad clínica rutinaria por los riesgos de la potencial transmisión derivados, principalmente, de la presencia de aerosoles de saliva en algunas de las prestaciones odontológicas (Pan et al., 2020). Tras la declaración de la pandemia por COVID-19 muchas de las atenciones dentales se han suspendido. Entre otras razones, porque las autoridades sanitarias han recomendado limitarlas solo a las atenciones de urgencias/emergencias odontológicas. Por ejemplo, en Chile en las Orientaciones para la Atención odontológica en Fase IV COVID-19 del Ministerio de Salud (Ministerio de Salud, 2020). Dichas atenciones también se han visto limitadas debido a la escasez de los elementos de protección personal (EPP) en distintos países durante los primeros meses de la pandemia (Pecchia et al., 2020). Por otro lado, las personas han dejado de asistir al dentista, ya sea por el temor que se ha generado, o como secuencia de la situación de confinamiento, recomendado u obligatorio, en la que gran parte de la población mundial ha sido expuesta.

Desde nuestro punto de vista, y como hemos señalado, estas razones podrían explicar el escaso reporte de lesiones de la mucosa oral en pacientes COVID-19 u otras manifestaciones orales. Especialmente, si consideramos que, en muchos entornos, son los odontólogos los profesionales más idóneos para evaluar clínicamente la salud oral de las personas.

Entre los síntomas orales que se han descrito en los pacientes COVID-19, están la ageusia (Vaira et al., 2020), la hipogeusia y la disgeusia (Vinayachandran \& Balasubramanian, 2020), Esto es de particular importancia porque, el receptor de la enzima convertidora de angiotensina 2, al que se une el SARS-CoV-2 para poder entrar en la célula huésped, se expresa altamente en las células epiteliales, especialmente en la lengua, en comparación con los tejidos orales o gingivales de la cavidad oral (Xu et al., 2020). Por otro lado, hasta mediados de junio de 2020 se han publicado solo cuatro reportes (Chaux-Bodard et al., 2020; Galván et al., 2020; Jimenez-Cauhe et al., 2020; Carreras-Presas et al., 2020) y además otro, que es el único que incluye el estudio histopatológico y que menciona un posible mecanismo etipatologénico (Soares et al., 2020). Todos esos reportes abordan una escasa casuística, lo que sugiere, por un lado, que algunas lesiones orales pudieran ser expresión clínica de la infección por SARS-CoV-2, y por otro, que los pacientes deban ser evaluados con un examen oral completo para diagnosticar adecuadamente la presencia posible de estas lesiones y relacionarlas con la COVID-19.

\footnotetext{
1 Área de Salud Pública del Instituto de Investigación en Ciencias Odontológicas, Facultad de Odontología, Universidad de Chile, Chile.

${ }^{2}$ Becario Postdoctoral en Unidad de Control de Tabaco, Instituto Catalán de Oncología, Barcelona, España.

${ }^{3}$ Departamento de Patología y Medicina Oral, Facultad de Odontología, Universidad de Chile, Chile.
} 
Carreras-Presas et al., reportaron el caso de un paciente que presentó una manifestación oral de aspecto herpetiforme, probablemente asociada a infección por SARS-CoV-2. Un atlas dermatológico muestra 4 fotografías intraorales de lesiones orales que presentaron 3 pacientes a quienes se les examinó la cavidad oral (Galván et al.). En recientes cartas al editor (Jimenez-Cauhe et al.) sobre lesiones dermatológicas en 4 pacientes, tres de los cuales presentaron lesiones en la mucosa oral.

El escaso número de reportes puede deberse a que las manifestaciones en mucosa oral son poco frecuentes, pero también puede haber sido favorecido porque muchos pacientes que han padecido la enfermedad, y eventualmente hayan tenido manifestaciones orales, no fueron evaluados por odontólogos, profesionales responsables del diagnóstico de estas lesiones.

En este contexto, concordamos con la reciente publicación de Soares et al., respecto a que cobra mayor relevancia incorporar rutinariamente la evaluación de signos y síntomas que se pueden presentar a nivel de la cavidad oral en pacientes que han sido diagnosticados positivos en el test de RT-PCR utilizado para el diagnóstico de infección por SARS-Cov-2. Ya sea para determinar si efectivamente, como se ha sugerido (Chaux-Bodard et al.), forma parte de la semiología de la COVID-19, o si, la presencia de manifestaciones orales como las descritas corresponden más bien a distintas entidades patológicas, que pudieran estar favorecidas por la respuesta inmune que desencadena el referido coronavirus.

Por ello, quienes suscribimos creemos necesario que la atención de salud de esos pacientes pueda contemplar la evaluación de su salud oral, por profesionales entrenados adecuadamente para ello. Además hacemos un llamado a que, como ya lo están haciendo algunos equipos de Atención Primaria de Salud (APS) de nuestro país, incorporen la evaluación de la salud oral en el seguimiento de las personas que han sido diagnosticadas como portadoras del SARS-CoV-2. Ello, teniendo en cuenta que la inserción histórica de los servicios odontológicos en la APS, representa una oportunidad comparativa respecto a otros entornos donde el sistema de salud no incluye servicios odontológicos.

Este manuscrito, en parte ha sido posible gracias al apoyo del Programa Beca Postdoctorado en el Extranjero de la Agencia Nacional de Investigación y Desarrollo (ANID), que financia la estadía postdoctoral de Marco Cornejo.

\section{REFERENCIAS BIBLIOGRÁFICAS}

Carreras-Presas, C. M.; Amaro Sánchez, J.; López-Sánchez, A. F.; JanéSalas, E. \& Somacarrera Pérez, M. L. Oral vesiculobullous lesions associated with SARS-CoV-2 infection. Oral Dis., 2020. DOI: https:/ /www. doi.org/10.1111/odi.13382

Chaux-Bodard, A. G.; Deneuve, S. \& Desoutter, A. Oral manifestation of Covid-19 as an inaugural symptom? J. Oral Med. Oral Surg., 26:18, 2020.

Galván Casas, C.; Català, A.; Carretero Hernández, G.; RodríguezJiménez, P.; Fernández-Nieto, D.; Rodríguez-Villa Lario, A.; Navarro Fernández, I.; Ruiz-Villaverde, R.; Falkenhain-López, D.; Llamas Velasco, M.; et al. Classification of the cutaneous manifestations of COVID -19: a rapid prospective nationwide consensus study in Spain with 375 cases. Br. J. Dermatol., 2020. DOI: https://www.doi.org/ 10.1111/bjd.19163

Guan, W. J.; Ni, Z. Y.; Hu, Y.; Liang, W. H.; Ou, C. Q.; He, J. X.; Liu, L.; Shan, H.; Lei, C. L.; Hui, D. S. C.; et al. Clinical characteristics of Coronavirus Disease 2019 in China. N. Engl. J. Med., 382(18):170820, 2020.

Jimenez-Cauhe, J.; Ortega-Quijano, D.; Carretero-Barrio, I.; Suarez-VaIle, A.; Saceda-Corralo, D.; Moreno-Garcia Del Real, C. \& FernandezNieto, D. Erythema multiforme-like eruption in patients with COVID19 infection: clinical and histological findings. Clin. Exp. Dermatol., 2020. DOI: https://www.doi.org/10.1111/ced.14281

Ministerio de Salud (MINSAL). Orientaciones para Atención Odontológica en Fase IV COVID-19. Santiago de Chile, Subsecretaría de Salud Pública División de Prevención y Control de Enfermedades, Departamento Salud Bucal, Ministerio de Salud, Gobierno de Chile, 2020. Disponible en: https://diprece.minsal.cl/wp-content/ uploads/2020/03/ ORIENTACIONES-ATENCION-ODONTOLOGICAS-COVID-19-.pdf

Pan, Y.; Liu, H.; Chu, C.; Li, X.; Liu, S. \& Lu, S. Transmission routes of SARS-CoV-2 and protective measures in dental clinics during the COVID-19 pandemic. Am. J. Dent., 33(3):129-34, 2020.

Pecchia, L.; Piaggio, D.; Maccaro, A.; Formisano, C. \& ladanza, E. The inadequacy of regulatory frameworks in time of crisis and in lowresource settings: personal protective equipment and COVID-19. Health Technol. (Berl.), 1-9, 2020. DOI: https://www. doi.org/10.1007/ s12553-020-00429-2

Soares, C. D.; de Carvalho, R. A.; de Carvalho, K. A.; de Carvalho, M. G. F. \& de Almeida, O. P. Letter to Editor: Oral lesions in a patient with Covid-19. Med. Oral Patol. Oral Cir. Bucal, DOI: https://www.doi.org/ 10.4317/medoral.24044

Vaira, L. A.; Salzano, G.; Deiana, G. \& De Riu, G. Anosmia and ageusia: common findings in COVID-19 patients. Laryngoscope, 130(7):1787, 2020.

Vinayachandran, D. \& Balasubramanian, S. Is gustatory impairment the first report of an oral manifestation in COVID-19? Oral Dis., 2020. DOI: https://www.doi.org/10.1111/odi.13371

Xu, H.; Zhong, L.; Deng, J.; Peng, J.; Dan, H.; Zeng, X.; Li, T. \& Chen, Q. High expression of ACE2 receptor of 2019-nCoV on the epithelial cells of oral mucosa. Int. J. Oral Sci., 12:8, 2020

Dirección para correspondencia:

Marco Cornejo Ovalle

Área de Salud Pública

Instituto de Investigación en Ciencias Odontológicas

Facultad de Odontología

Universidad de Chile

Santiago - CHILE

Email: mcornejo@odontologia.uchile.cl

Recibido : 15-06-2020

Aceptado: 18-06-2020 